\title{
Survival analysis: A consumer-friendly method to estimate the optimum sucrose level in probiotic petit suisse
}

\author{
E. A. Esmerino, ${ }^{*}$ J. A. Paixão, ${ }^{*}$ A. G. Cruz, $†$ L. Garitta, $\ddagger$ G. Hough, $\ddagger$ and H. M. A. Bolini* \\ *Faculty of Food Engineering, State University of Campinas, Campinas, São Paulo, Brazil 13083-862 \\ †Federal Institute of Education, Science and Technology of Rio de Janeiro, Rio de Janeiro, Brazil 20260-100 \\ ‡Departamento de Evaluación Sensorial de Alimentos, Instituto Superior Experimental de Tecnología Alimentaria, Buenos Aires, Argentina 6500
}

\section{ABSTRACT}

For years, just-about-right (JAR) scales have been among the most used techniques to obtain sensory information about consumer perception, but recently, some researchers have harshly criticized the technique. The present study aimed to apply survival analysis to estimate the optimum sucrose concentration in probiotic petit suisse cheese and compare the survival analysis to JAR scales to verify which technique more accurately predicted the optimum sucrose concentration according to consumer acceptability. Two panels of consumers (total $=170$ ) performed affective tests to determine the optimal concentration of sucrose in probiotic petit suisse using 2 different methods of analysis: JAR scales $(\mathrm{n}=85)$ and survival analysis ( $\mathrm{n}$ $=85)$. Then an acceptance test was conducted using naïve consumers $(\mathrm{n}=100)$ between 18 and $60 \mathrm{yr}$ old, with 2 samples of petit suisse, one with the ideal sucrose determined by JAR scales and the other with the ideal sucrose content determined by survival analysis, to determine which formulation was in accordance with consumer acceptability. The results indicate that the 2 sensory methods were equally effective in predicting the optimum sucrose level in probiotic petit suisse cheese, and no significant differences were detected in any of the characteristics related to liking evaluated. However, survival analysis has important advantages over the JAR scales. Survival analysis has shown the potential to be an advantageous tool for dairy companies because it was able to accurately predict the optimum sucrose content in a consumer-friendly way and was also practical for researchers because experimental sensory work is simpler and has been shown to be more cost effective than JAR scales without losses of consumer acceptability.

Key words: survival analysis, just-about-right scale, optimum sucrose level, petit suisse cheese

Received March 30, 2015.

Accepted June 25, 2015.

${ }^{1}$ Corresponding author: erick.almeida@hotmail.com

\section{INTRODUCTION}

Petit suisse is an unripened, unsalted, smooth, and creamy cheese with a texture closer to a very thick yogurt than a typical cheese. It is consumed worldwide and is one of the most favored French soft cheeses (Ramírez-Santiago et al., 2012). It is made from pasteurized cow milk and has a fat content of approximately $18.8 \%$ on a DM basis (Prudencio et al., 2008). Petit suisse is frequently combined with fruit and consumed as a dessert and is designed to target children as consumers, although it is well accepted by all age groups (Carderelli et al., 2008; Matias et al., 2014).

Because the field of nutrition is moving toward the use of foods to promote better health and well-being, functional foods have become increasingly more important for consumers (Carderelli et al., 2008). Probiotic petit suisse cheeses were shown to be very promising as a functional food because their dense matrix and relatively high fat content showed appropriate conditions for maintaining the viability of probiotic microorganisms throughout storage until consumption (Maruyama et al., 2006; Carderelli et al., 2008; Cruz et al., 2010).

Consumer research is essential in the development and marketing of new products, reformulation of existing products, optimization of manufacturing processes, and establishment of specifications in quality-control systems (Van Kleef, 2006). Because consumer opinion cannot be ignored, food companies need information about how consumers perceive food products, including their sensory attributes and how they can be modified or adapted to increase the product's acceptability (Barrios and Costell, 2004).

In this sense, just-about-right (JAR) scales are among the most used techniques to obtain sensory information about consumer perception of a product mainly because they are convenient to use (Popper et al., 2004; Ares et al., 2009; Gaze et al., 2015). The JAR scales have been used to determine the optimum level of certain ingredients in various food matrices (Esmerino et al., 2013; Menis et al., 2013; Morais et al., 2014; de Oliveira Rocha and Bolini, 2015). 
Despite the widespread use of JAR scales, some researchers have criticized their use because they combine the measurements of sensory attribute intensity and consumer acceptability, and it has been suggested that JAR scales should not take the place of traditional experimental product design (Moskowitz and Hartmann, 2008). Others have affirmed that JAR scaling is a demanding task for naïve consumers because these ratings involve at least 3 important decisions: (1) identification of the sensory intensity, (2) participants' ideal point position, and (3) comparison of the variation between the perceived intensity and ideal imagined point (Gacula et al., 2008). Furthermore, certain attributes can present health bias when optimum levels are analyzed by JAR scales. It occurs when some attributes that can carry negative connotations, as can occur with sweetness, negatively affect the sweetness rating. A very sweet product can be seen as unhealthy by consumers, and their results can be wrongly influenced by personal concepts do not representing the reality of consumer preference (Epler et al., 1988).

An interesting and alternative option to solve this point seems to be the survival analysis methodology. This method has been developed to evaluate the time until an event of interest, often called the survival time, by taking into account the presence of censored data (Garitta et al., 2006). This event of interest can be replaced by other variables in the system under study, including product shelf-life or sensory defects or, as in the present work, ingredient concentration.

In food science, survival analysis methods have been primarily applied to shelf-life estimation of foods, where the event of interest was substituted with storage time (Hough et al., 2003, 2004). Then, some studies replaced time with the concentration of other variables. Sensory defects in UHT milk, such as acid, caramel, doneness, and dark color, were assessed to determine how high they could be before a consumer rejects the product (Giménez et al., 2012). In a recent study, survival analysis was applied to equally sweet determinations of some sweeteners (Alcaire et al., 2014).

Some variations on the technological processes or modifications of food formulations may cause important and perceptible effects on sensory characteristics. With this perspective, sensory analysis must be carefully conducted to evaluate the maximum limits of the alteration that is supported by the consumer before the product rejection process occurs (Giménez et al., 2012).

Thus, the aim of this work was (1) to apply the survival analysis method to estimate the optimum sucrose concentration according to naïve consumers' opinions and (2) to compare with standard JAR scales to predict the optimum sucrose level based on acceptance testing of strawberry-flavored probiotic petit suisse samples.

\section{MATERIALS AND METHODS}

\section{Probiotic Petit Suisse Cheese Processing}

Petit suisse cheese was prepared according to the methodology described by Esmerino et al. (2013) using probiotic cultures of Lactobacillus acidophilus and Bifidobacterium lactis (Danisco, São Paulo, Brazil). The mass (quark cheese) was obtained by heating pasteurized milk to 37 to $38^{\circ} \mathrm{C}$, transferring it to isothermal containers and then adding the starter and probiotic cultures by direct inoculation followed by homogenization. The containers with the inoculated milk were maintained at $37^{\circ} \mathrm{C}$ until the $\mathrm{pH}$ level was between 6.3 to 6.5 ; then, the rennet was added, re-homogenized and maintained at $37^{\circ} \mathrm{C}$ until a curd was formed with a $\mathrm{pH}$ level between 5.6 to 5.8. The curd was carefully cut into cubes that were placed in sterile cloth sacks to drain off the whey at a temperature of $10^{\circ} \mathrm{C}$ for $15 \mathrm{~h}$. After drainage, the quark cheese was placed in sterile containers and stored at $4^{\circ} \mathrm{C}$ until mixed with the different sucrose levels $(7.5,11,14.5,18$, and $21.5 \% \mathrm{wt} / \mathrm{wt})$. Ingredients were added and homogenized in a multi-processor for 30 min (Estephan rpm 1750/3500, Geiger, Pinhais, Paraná, Brazil) until a consistent mass was obtained. Appropriate containers were filled with probiotic petit suisse cheeses and cheeses were maintained under refrigeration at $4^{\circ} \mathrm{C}$ until the day of analysis. Then, these samples with different concentrations of sucrose were used to determine the optimum sucrose concentration for both analysis methods.

Finally, after the determination of the optimum sucrose levels for each methodology, petit suisse cheeses were prepared in accordance with Esmerino et al. (2013), but in the sweetening stage, the quark cheese was separated into 2 parts: one was mixed with the sucrose levels that were determined by survival analysis and the other with the sucrose levels that were determined by the JAR scales.

\section{Sensory Evaluations}

All of the sensory tests were conducted in individual booths conforming to the International Standards (ISO, 1988) and were equipped with FIZZ Network Sensory Software (Biosystemes, Couternon, France), adequate illumination, and controlled temperature (between 22 and $25^{\circ} \mathrm{C}$ ) in the Sensory Analysis Laboratory of the Department of Food and Nutrition of the State University of Campinas, Brazil. Samples were served at $10 \pm$ $2^{\circ} \mathrm{C}$ in disposable $50-\mathrm{mL}$ plastic cups coded with 3 -digit random numbers.

The presentation was monadic and balanced to minimize order and carryover effects (Macfie et al., 1989). 
Consumers were also asked to rinse their palates with water and crackers to avoid contrast errors between samples.

Approval for the study was obtained from the Ethics Committee of the University of Campinas, and written consent was given by all volunteers.

\section{Survival Analysis}

Eighty-five consumers, undergraduate and graduate students and employees on the State University of Campinas campus, who consume petit suisse cheese at least once a week and whose ages ranged between 18 to $55 \mathrm{yr}$ old ( $60 \%$ female), were recruited. Aliquots of 20 to $30 \mathrm{~g}$ of each strawberry-flavored probiotic petit suisse cheese $(7.5,11,14.5,18$, and $21.5 \% \mathrm{wt} / \mathrm{wt})$ were presented to each panelist.

After receiving each sample, consumers were requested to answer the following question: "Considering the sweetness of this sample, if the product was available for consumption, would you consume it? Yes or No?" It was explained that the question should take into account just the sweetness perceived in each of the samples presented and the only answers were the 2 above-mentioned options. This information was sufficient to model the probability of consumers rejecting the products with different concentrations (Hough et al., 2013).

If a consumer accepted the sample with a concentration of $11 \%$ and rejected the sample with a concentration of $14.5 \%$, the exact concentration of rejection could be any amount between 11 and $14.5 \%$. This is defined as interval censoring. When a consumer rejects the sample with a concentration of 7.5 , the rejection concentration is $\leq 7.5$; this is called left censoring. If the consumer accepted all of the concentrations, rejection could occur for a concentration $\geq 21.5 \%$, and the data were right censored (Giménez et al., 2007).

Defining a random variable $C$ as the sucrose level (as measured by consumers) at which the consumer rejects the sample, the rejection function $F(C)$ can be defined as the probability (percentage) of a consumer rejecting a sample with a $C \%$ of sucrose equal to or more than a product with $7.5 \%$. Choosing a lognormal distribution for $C$ (Hough et al., 2004), the rejection function is given by

$$
F(c)=1-\mathrm{S}_{\mathrm{sev}}\left[\frac{\ln (\mathrm{c})-\mu}{\sigma}\right]
$$

where $\mathrm{S}_{\mathrm{sev}}$ is the survival function of the smallest extreme value distribution and $\mu$ and $\sigma$ are the model's parameters. The parameters of the model are obtained by maximizing the likelihood function, which is a mathematical expression that describes the joint probability of obtaining the data that were observed on the subjects in the study as a function of the unknown parameters of the model being considered. Once the likelihood function has been established for a given model, specialized software can be used to estimate the parameters ( $\mu$ and $\sigma)$ that maximize the likelihood function for the given experimental data.

For more details on the likelihood and survival functions, see Hough et al. (2004) and Alcaire et al. (2014).

\section{JAR Scales}

The optimum sucrose concentration that was added to the strawberry-flavored probiotic petit suisse cheese was also determined by an affective test using JAR scales (Meilgaard et al., 2004). Eighty-five consumers (approximately 90\% of consumers performed the survival analysis task), recruited from undergraduate and graduate students and employees on the university campus, who consume petit suisse cheese at least once a week, with an age range between 18 and $55 \mathrm{yr}$ old, took part in the test. Consumers evaluated aliquots of 20 to $30 \mathrm{~g}$ of each $(7.5,11,14.5,18$, and $21.5 \% \mathrm{w} / \mathrm{w})$ strawberry-flavored probiotic petit suisse cheese using a 9-cm continuous scale that was anchored at the left with "extremely less sweet," at the right by "extremely too sweet," and at the central point with a JAR concentration for this product. The use of 9-pt JAR scale is widely used in sensory analysis and it has been successfully employed in several studies (Menis et al., 2013; de Oliveira Rocha and Bolini, 2015).

\section{Consumer Acceptance Test}

The sensory acceptance test was conducted using naïve panelists who consume petit suisse cheese at least once a week ( $\mathrm{n}=100$; Stone et al., 2012), with an age range between 18 and 60 yr old ( $55 \%$ women). Most of the consumers $(85 \%)$ did not participate in the preliminary sensory studies.

The sensory test was performed in one session, where aliquots of 20 to $30 \mathrm{~g}$ of both strawberry-flavored probiotic petit suisse cheese samples, each one with optimum concentrations determined by survival analysis and JAR scales, were served. Prior to tasting, the subjects were instructed in the use of the 9 -cm hedonic scale, which was labeled from $1=$ dislike extremely to $9=$ like extremely (Morais et al., 2014; de Oliveira Rocha and Bolini, 2015). The consumers were asked to assess the following attributes: appearance, aroma, flavor, texture, and overall acceptability. 


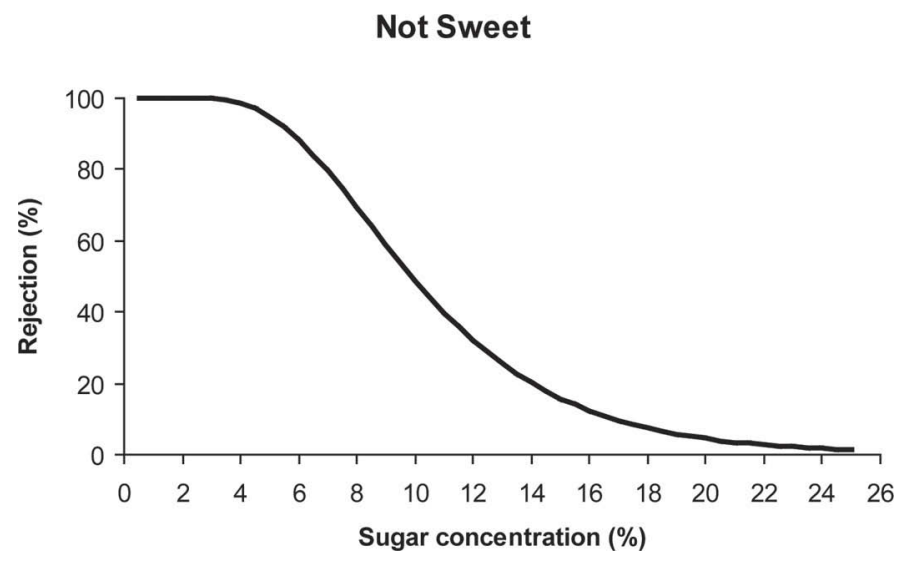

Figure 1. Percentage of rejection of probiotic petit suisse cheese by consumers versus the concentration of sucrose in not sweet function in survival analysis.

\section{Statistical Analysis}

Data that were obtained in the survival analysis were collected and converted by FIZZ Network Sensory Software. Calculations to estimate the parameters $\mu$ and $\sigma$ and the rejection functions were performed using procedures found in SAS (SAS Institute Inc., Cary, NC) and XLSTAT version 2011 (Addinsoft, Paris, France; Hough et al., 2004).

The results from the JAR scale were also collected and converted using FIZZ Software. They were analyzed by a simple linear regression analysis to determine the exact sucrose concentration that was considered just about right by consumers and visualized by a histogram bar as suggested by Vickers (1988) in Microsoft Office Excel 2007 (version 97-2003) for Windows.

To compare the accuracy of both the determination of the optimum concentration of sucrose with hedonic assessments and consumer acceptability, the data obtained in the consumer acceptance test were analyzed by a $t$-test using SPSS v. 8.0 (SPSS Inc., Chicago, IL) software for Windows. The probability level of $P<$ 0.05 was considered significant.

\section{RESULTS AND DISCUSSION}

\section{Determination of the Optimum Sucrose Concentration Using Survival Analysis}

The sucrose concentration that was considered optimum by the consumer was defined as the random variable $C$, assuming that $C$ was absolutely continuous with a distribution function of $F$. In shelf-life studies with 1 event model (appropriate to not appropriate), samples with different storage times are presented to consumers, and the survival function $\mathrm{S}(\mathrm{t})$ can be defined as the probability of a consumer accepting a product that was beyond time (Hough et al., 2003). In 2 event models (from not sweet enough to appropriate and from appropriate to too sweet), 2 probability functions must exist (Klein and Moeschberger, 1997): $\mathrm{S}_{\mathrm{L}}$ (too little sucrose) and $\mathrm{S}_{\mathrm{M}}$ (too much sucrose).

Because no statistical test is available to compare the quality of the correlation between different parametric models used for the data in an interval of censure, it was necessary to evaluate visually how the parametric models fit the nonparametric models to choose the most adequate model (Cruz et al., 2010). The following distributions were considered: smallest extreme value, normal, logistic, Weibull, log normal, and log logistic (Hough et al., 2003). However, the log normal distribution has been shown to be the most adequate for the data from both events.

The rejection percentage according to the sucrose concentration of the strawberry-flavored probiotic petit suisse cheeses can be observed in Figures 1 and 2 . Consumers' percent rejections that are most commonly used are 10, 25, and 50\% (Hough et al., 2003, 2004; Gambaro et al., 2006). However, Garitta et al. (2006) suggested that in the survival analysis of a 2-event model, the optimum concentration could be determined by finding the minimum value of the curve, which is the result of the addition of both event rejection curves.

The overlapping of the 2 event curves of interest can be observed in Figure 3, where it is possible to visualize the mid-point of the curves and determine the optimum sucrose concentration that leads to the minimum percent consumer rejection. Table 1 shows the parameters used to calculate the optimum sucrose concentration added to the strawberry-flavored probiotic petit suisse cheese. The total percentage of rejection at the optimum sucrose concentration, the percentage of rejection

\section{Too Sweet}

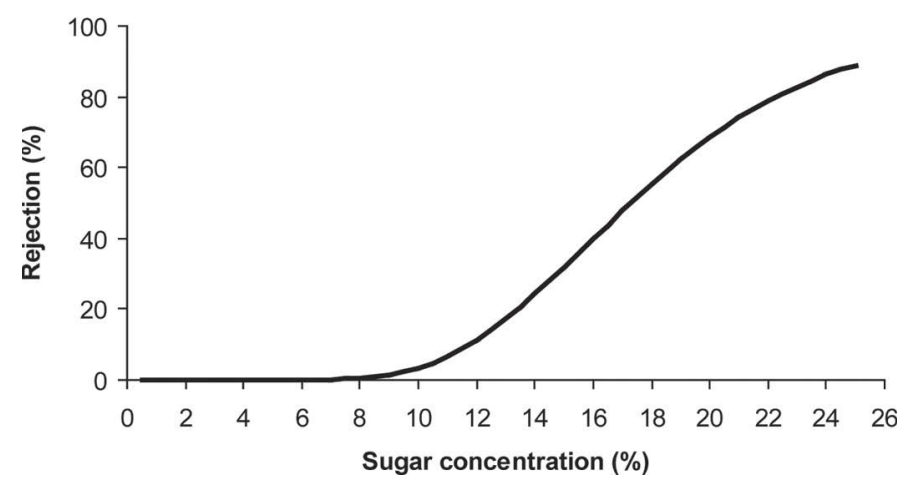

Figure 2. Percentage of rejection of probiotic petit suisse cheese by consumers versus the concentration of sucrose in too sweet function in survival analysis. 
Table 1. Optimum sucrose concentration $\pm 95 \%$ confidence intervals and probability of a subject rejecting these concentrations due to them not being sweet enough or too sweet obtained using survival analysis

\begin{tabular}{|c|c|c|c|c|c|}
\hline $\begin{array}{l}\text { Optimum sucrose } \\
\text { concentrations } \pm 95 \% \\
\text { confidence limits }(\%)\end{array}$ & $\begin{array}{l}\text { Total percentage } \\
\text { of rejection due } \\
\text { to optimal }(\%)\end{array}$ & $\begin{array}{l}\% \text { Probability } \\
\text { of rejection due to } \\
\text { not sweet enough }\end{array}$ & $\begin{array}{l}\% \text { Probability } \\
\text { of rejection } \\
\text { due to too sweet }\end{array}$ & $\begin{array}{l}\text { SD for being } \\
\text { not too sweet }\end{array}$ & $\begin{array}{l}\text { SD for being } \\
\text { too sweet }\end{array}$ \\
\hline
\end{tabular}

considering the optimum concentration as not sweet enough, the percentage of rejection considering the optimum concentration as too sweet, and the standard deviations for all of them can be observed.

The results indicated that the optimum sucrose concentration to be added to probiotic petit suisse cheese was $12.74 \pm 1.09 \mathrm{~g} / 100 \mathrm{~g}$ of product (with $95 \%$ confidence). Because the standard deviation was approximately 1.09 , a sucrose concentration of $12.70 \%$ was adopted for subsequent studies. For this concentration, the rejection percentage by consumers was $42 \%$, of which $27 \%$ was considered the optimum concentration to be not sweet enough and $15.6 \%$ considered it too sweet. Considering the high percentage of rejection, 2 types of consumers were assumed: those who liked their petit suisse cheeses less sweet and those who preferred them sweeter. Thereby, among the 85 consumers who took part in the evaluation, it was possible to observe some heterogeneity in the population, and the data obtained must be carefully analyzed before making a final decision about the formulation. We suggest that for further studies, segmentation of the consumers may be considered in the survival analysis method.

According to Garitta et al. (2006), judgments carried out using survival analysis are considered to show greater agreement with the reality of consumer choices. The JAR scales are a demanding test for consumers because they have to perceive the attribute intensity,

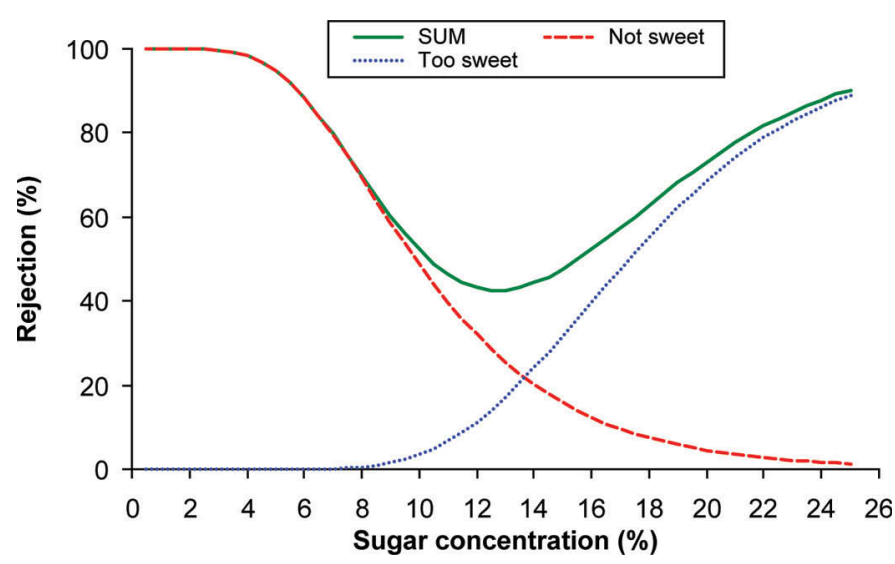

Figure 3. Optimal concentration of sucrose (overlay for both curves indicates rejection for not sweet and too sweet) in survival analysis. Color version available online. find an appropriate location for the ideal point, and mentally compare the difference between perceived intensity and their ideal point.

According to consumer feedback, survival analysis is consumer friendly and easier than JAR scales. Some consumers reported practicality and that it was less time consuming to solve the task, which was also observed by researchers. This can be mainly explained by capture of answers. Although performance intensity scales are used in JAR, in survival analysis only responses related to consumption, limited to the answers yes or no, are requested. When performing survival analysis, consumers are not pressured to evaluate the intensity of an attribute, they indicate only if they would consume or purchase the product at different concentrations (Hough et al., 2003, 2004). It sounds more natural to consumers because making decisions is part of their consumption routine, making their results more reliable; thus, survival analysis may more clearly represent the expectations of consumers with respect to a product.

\section{Determination of the Sucrose Concentration Using JAR Scales}

According to the evaluation of consumers, a significant difference at a $5 \%$ probability by an $F$-test can be observed between the samples that were sweetened with different concentrations of sucrose. Therefore, a regression model was adjusted to relate the JAR sweetness with the sucrose concentrations of processed probiotic petit suisse samples. The linear model showed a good data fit (coefficient of determination of 0.92). The regression equation (Figure 4) allowed for the determination of the JAR sweetness of the probiotic petit suisse according to consumer perceptions. The amount of sucrose to be added to the probiotic petit suisse was determined to be $15.17 \%$, but for subsequent experiments, a sucrose concentration of $15.20 \%$ was used.

The differences found between concentrations can be explained by the different methodological aspects and type of data involved. The JAR scale uses continuous scales (perceived as a difficult task for naïve consumers) resulting in parametric data. Survival analysis has the big advantage of being easier to apply, easier to be understood by consumers, and also easier on the 
Table 2. Mean values of liking attributes evaluated by consumers in acceptance test for probiotic petit suisse cheese samples with different concentrations of sucrose

\begin{tabular}{|c|c|c|c|c|c|}
\hline Sample & Appearance & Aroma & Flavor & Texture & $\begin{array}{c}\text { Overall } \\
\text { liking }\end{array}$ \\
\hline Survival analysis & $6.41^{\mathrm{a}}$ & $6.67^{\mathrm{a}}$ & $6.35^{\mathrm{a}}$ & $6.61^{\mathrm{a}}$ & $6.75^{\mathrm{a}}$ \\
\hline $\mathrm{JAR}^{1}$ scales & $6.54^{\mathrm{a}}$ & $6.21^{\mathrm{a}}$ & $6.44^{\mathrm{a}}$ & $6.76^{\mathrm{a}}$ & $6.54^{\mathrm{a}}$ \\
\hline
\end{tabular}

obtaining results step. Because of the nonparametric nature of data, the results are easily and quickly collected and the experimental work is optimized, becoming more practical for research.

Different values for the optimum sucrose concentration were found in different types of food, including mango nectar, $7.5 \mathrm{~g} / 100 \mathrm{~g}$ (Cadena and Bolini, 2012), passion fruit juice $9.4 \mathrm{~g} / 100 \mathrm{~g}$ (de Oliveira Rocha and Bolini, 2015), and other dairy products, such as chocolate milk (Paixão et al., 2014). De Souza et al. (2011), in a similar study to establish the exact sucrose concentration in conventional petit suisse, found that the JAR concentration of sucrose to be added was $17 \%$. The differences were expected and are most likely a result of the interactions between sweeteners, product components, and probiotic metabolites (Esmerino et al., 2013).

The differences in the exact concentration of similar products illustrate the important of performing sensory analysis on each product (de Oliveira Rocha and Bolini, 2015).

\section{Consumer Acceptance Test}

Because one of the objectives of this study was to verify which technique is more accurate in predicting the optimum sucrose level that is close to consumers' expectations about probiotic petit suisse, an acceptance test was applied on 2 different samples, one with $15.2 \%$ sucrose, as determined by JAR scales, and the other with $12.7 \%$ sucrose, as established by survival analysis.

Flavor is a product of multisensory interactions but most notably those of taste, smell, and the trigeminal system, and it has a major role in determining the acceptability of foods and beverages (Tournier et al., 2009; Small, 2012). From evaluating the flavor, sensations linked to gustatory sense as sweet, sour, salty, bitter, and savory can be indirectly evaluated (Prescott, 2015; Spence, 2015). In this sense, we chose to not separately evaluate the liking of sweetness.

The samples came from the same batch and differed only in their sucrose content. Aliquots of 20 to $30 \mathrm{~g}$ of both strawberry-flavored probiotic petit suisse cheeses were evaluated by $100(\mathrm{n}=100)$ panelists $(55 \%$ women $)$ who consume petit suisse once per week. The mean hedonic scores attributed to the 2 evaluated samples with the sucrose content established using survival analysis and JAR scales can be observed in Table 2 .

Variations in the sucrose content can modify intrinsic interactions among food components and may be reflected and clearly perceptible in sensory attributes of color, aroma, flavor, texture; these variations could lower consumer acceptance (Arancibia et al., 2013).

The mean scores obtained from samples containing the sucrose concentration as determined by the JAR scale $(15.2 \% \mathrm{wt} / \mathrm{wt})$ were slightly higher for liking of appearance, flavor, and texture, with mean values of $6.54,6.44$, and 6.76, respectively. Products made with less sucrose tend to be less viscous and some losses in texture can be observed, but in our findings, no significant differences $(P \geq 0.05)$ were found in any of the above-mentioned characteristics related to liking compared with the sample sweetened with $12.7 \%$ of sucrose, as determined by survival analysis. Samples containing $12.7 \%$ of sucrose (survival analysis) showed slightly higher mean aroma (6.67) and overall liking (6.75) scores, but no significant differences $(P \geq 0.05)$ were observed.

Lethuaut et al. (2005) reported that different sucrose concentrations might influence the perception of sweetness and flavor. Soukoulis and Tzia (2009) dem-

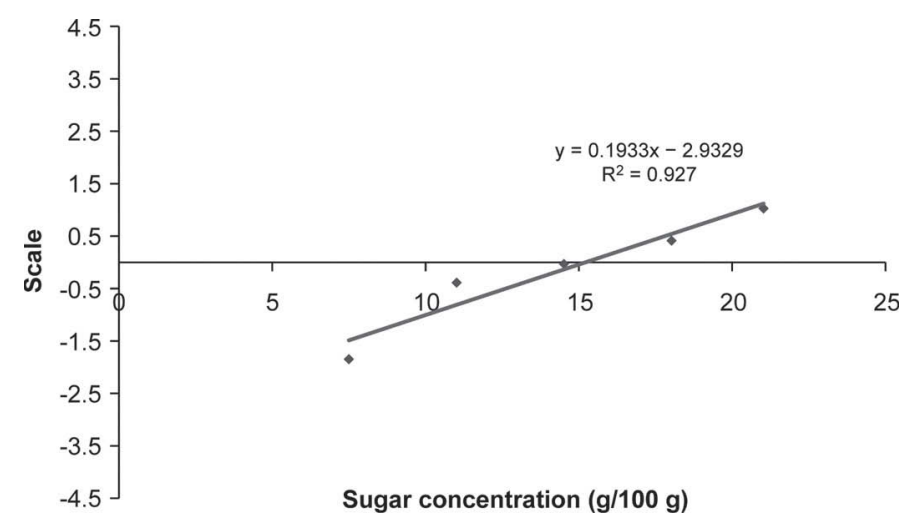

Figure 4. Equation of the line obtained in just-about-right test to determine the ideal concentration of sucrose to be added to the petit suisse probiotic strawberry flavor. 
onstrated that in a chocolate ice cream, the partial replacement of sucrose by different sweetener compounds might affect the flavor that is released and the texture. The addition of sucrose may induce an increase of perceived aroma intensity such as vanilla intensity (Kuo et al., 1993), almond intensity or various fruity aroma intensities (Lethuaut et al., 2005). Even an increase of viscosity (semi-solid systems) or hardness (gel systems) was consistently found to reduce both perceived taste intensity (Soukoulis et al., 2008) and perceived aroma intensity (Boland et al., 2006).

In contrast with the above-mentioned studies, no significant differences were found in the present work when comparing samples from both methodologies for any of characteristics related to liking that were evaluated in the acceptance test. The different concentrations of sucrose that were established by survival analysis and the JAR scale were shown to be statistically indifferent $(P$ $\geq 0.05$ ) with regard to producing changes in consumer perception. However, further studies should evaluate a greater number of consumers because differences can be present with respect to the sweetness content preference for each consumer.

Results from the current work were shown to be in accordance with the study from Boeneke et al. (2006). They investigated the effects of sucrose replacement by sweeteners on a dairy-based espresso beverage that was manufactured with an ice cream mix. Their findings indicated that the trained panel found no significant differences in viscosity and color intensity between samples with $100 \%$ sucrose and a 50:50 blended sweetener (sugar and corn sweetener). No significant differences were detected between samples with $100 \%$ sucrose and a 50:50 blended sweetener for likeability attributes, such as overall flavor, body/texture, and appearance.

Given the variability of results in the mentioned studies, the effect of sucrose replacement on some sensory attributes was indicated to be food matrix dependent. Variations in the sucrose levels can positively or negatively affect a product, but in the present work, it has been shown to cause no effect on consumer acceptance of strawberry-flavored probiotic petit suisse.

According to the results, 2 suggestions can be highlighted: (1) although samples have been formulated with different concentrations of sucrose, sensory changes could have happened but were not able to generate sensory losses in consumer acceptance and both samples were equally well accepted by consumers, or (2) the 2 concentrations determined by JAR scale and survival analysis were not able to produce perceptible sensory changes and the consumers evaluated the samples in the same way.

A few studies have investigated sucrose replacement in the petit suisse matrix; however, data regarding how this substitution can affect the sensory quality and overall liking of the product are still limited (De Souza et al., 2011; Esmerino et al., 2013). Because some public health agencies desire for countries to commit to a dialog with food companies to reduce sugars in the composition of their products, investigations of the effect of sugar replacement on consumer acceptance may increase and should be accurately conducted. In this sense, this study highlights the importance of carefully conducted sensory analysis using appropriate methods that adequately indicate the perception of consumers.

\section{CONCLUSIONS}

Our findings indicated that when comparing the performance of 2 sensory methods, survival analysis and JAR scales, both methodologies were similarly effective in predicting the optimal concentration of sucrose for probiotic petit suisse cheese. The 2 methods provided similar indications of the amount of sucrose to be added to the product, and the consumer acceptance test confirmed that the 2 optimal formulations were equally liked with no significant differences for any of characteristics related to liking that were investigated. However, we observed that survival analysis presents important advantages over the JAR scales. Survival analysis proved to be a consumer-friendly task that was easier to perform than JAR scales and was more practical for researchers. It was also able to provide a reduced sugar content of probiotic petit suisse cheeses without changes or losses in sensory quality or acceptance and in a more cost-effective way. Although it needs to be studied further, survival analysis has shown potential as an advantageous tool for dairy companies because it is not restricted to sucrose and petit suisse cheese but could be applied to a wide range of products and ingredients.

\section{ACKNOWLEDGMENTS}

We acknowledge CNPq (Conselho Nacional de DEsenvolvimento Cientifico e Tecnologico - Brazil) and CAPES (Coordenacao de Aperfeicoamento de Pessoal de Nivel Superior - Brazil) for financial support.

\section{REFERENCES}

Alcaire, F., S. Zorn, R. S. Cadena, L. Antúnez, L. Vidal, A. Gimenez, and G. Ares. 2014. Application of survival analysis to estimate equivalent sweet concentration of low-calorie sweeteners in orange juice. J. Sens. Stud. 29:474-479.

Arancibia, C., S. Bayarri, and E. Costell. 2013. Impact of structural differences on perceived sweetness in semisolid dairy matrices. J. Texture Stud. 44:346-356.

Ares, G., C. Barreiro, and A. Gimenez. 2009. Comparison of attribute liking to evaluate the adequacy of sensory attributes of milk desserts. J. Sens. Stud. 24:664-676. 
Barrios, E. X., and E. Costell. 2004. Use of methods of research into consumers' opinions and attitudes in food research. Food Sci. Technol. Int. 10:359-371.

Boeneke, C. A., J. U. McGregor, and K. J. Aryana. 2006. The effect of sweeteners on the acceptability of dairy-based espresso beverages. Int. J. Dairy Technol. 59:12-17.

Boland, A. B., C. M. Delahunty, and S. M. Van Ruth. 2006. Influence of the texture of gelatin gels and pectin gels on strawberry flavour release and perception. Food Chem. 96:452-460.

Cadena, R. S., and H. M. A. Bolini. 2012. Ideal and relative sweetness of high intensity sweeteners in mango nectar. Int. J. Food Sci. Technol. 47:991-996.

Cardarelli, R. H., F. C. A. Buriti, I. A. Castro, and S. M. I. Saad. 2008. Inulin and oligofructose improve sensory quality and increase the probiotic viable count in potentially synbiotic petit-suisse cheese. LWT Food Sci. Technol. (Campinas.) 41:1037-1046.

Cruz, A. G., E. H. M. Walter, R. S. Cadena, J. A. F. Faria, H. M. A. Bolini, H. P. Pinheiro, and A. S. Sant'Ana. 2010. Survival analysis methodology to predict the shelf-life of probiotic flavored yogurt. Food Res. Int. 43:1444-1448.

de Oliveira Rocha, I. F., and H. M. A. Bolini. 2015. Different sweeteners in passion fruit juice: Ideal and equivalent sweetness. LWT Food Sci. Technol. (Campinas.) 62:861-867.

De Souza, V. R., A. C. M. Pinheiro, J. D. S. Carneiro, S. M. Pinto, L. R. Abreu, and C. C. Menezes. 2011. Analysis of various sweeteners in petit Suisse cheese: Determination of the ideal and equivalent sweetness. J. Sens. Stud. 26:339-345.

Epler, S., E. Chambers, and K. E. Kemp. 1988. Hedonic scales are a better predictor than just-about-right scales of optimal sweetness in lemonade. J. Sens. Stud. 13:191-197.

Esmerino, E. A., A. G. Cruz, E. P. R. Pereira, J. B. Rodrigues, J. A. F. Faria, and H. M. A. Bolini. 2013. The influence of sweeteners in probiotic Petit suisse cheese in concentrations equivalent to that of sucrose. J. Dairy Sci. 96:5512-5521.

Gacula, M., P. Mohan, J. Faller, L. Pollack, and H. R. Moskowitz. 2008. Questionnaire practice: What happens when the jar scale is placed between two "overall" acceptance scales? J. Sens. Stud. 23:136-147.

Gambaro, A., G. Ares, and A. Gimenez. 2006. Shelf-life estimation of apple baby food. J. Sens. Stud. 21:101-111.

Garitta, L., C. Serrat, G. Hough, and A. V. Curia. 2006. Determination of optimum concentrations of a food ingredient using survival analysis statistics. J. Food Sci. 71:526-532.

Gaze, L. V., B. R. Oliveira, L. L. Ferrao, D. Granato, R. N. Cavalcanti, C. A. Conte Júnior, A. G. Cruz, and M. Q. Freitas. 2015. Preference mapping of dulce de leche commercialized in Brazilian markets. J. Dairy Sci. 98:1443-1454.

Giménez, A., F. Ares, and G. Ares. 2012. Sensory shelf-life estimation: A review of current methodological approaches. Food Res. Int. 49:311-325.

Giménez, A., P. Varela, A. Salvador, G. Ares, S. Fiszman, and L. Garitta. 2007. Shelf life estimation of brown pan bread: A consumer approach. Food Qual. Prefer. 18:196-204.

Hough, G., L. Garitta, and R. Sanchez. 2004. Determination of consumer acceptance limits to sensory defects using survival analysis. Food Qual. Prefer. 15:729-734.

Hough, G., K. Langohr, G. Gomez, and A. M. L. Curia. 2003. Survival analysis applied to sensory shelf life of foods. J. Food Sci. 68:359-362.

Hough, G., L. Methven, and H. T. Lawless. 2013. Survival analysis statistics applied to threshold data obtained from the ascending forced-choice method of limits. J. Sens. Stud. 28:414-421.

ISO. 1988. Sensory analysis-General guidance for the design of test rooms. ISO 8589. International Organization for Standardization, Geneva, Switzerland.

Klein, J. P., and M. K. Moeschberger. 1997. Censoring and truncation. Pages 55-82 in Survival Analysis, Techniques for Censored and Truncated Data. Springer Verlag, New York, NY.

Kuo, Y., R. M. Pangborn, and A. C. Noble. 1993. Temporal patterns of nasal, oral, and retronasal perception of citral and vanillin and interaction of these odourants with selected tastants. Int. J. Food Sci. Technol. 28:127-137.

Lethuaut, L., C. Brossard, A. Meynier, F. Rousseau, G. Llamas, and B. Bousseau. 2005. Sweetness and aroma perceptions in dairy desserts varying in sucrose and aroma levels and in textural agent. Int. Dairy J. 15:485-493.

Macfie, H. J., N. Bratchel, K. Greenhoff, and L. Vallis. 1989. Designs to balance the effect of order of presentation and first-order carryover effects in hall tests. J. Sens. Stud. 4:129-148.

Maruyama, L. Y., H. R. Cardarelli, F. C. A. Buriti, and S. M. I. Saad. 2006. Textura instrumental de queijo petit-suisse potencialmente probiótico: Influência de diferentes combinações de gomas. Cien. Tecnol. Alim. 26:386-393.

Matias, N. S., R. Bedani, I. A. Castro, and S. M. I. Saad. 2014. A probiotic soy-based innovative product as an alternative to petitsuisse cheese. LWT Food Sci. Technol. (Campinas) 59:411-417.

Meilgard, M. T., G. V. Civille, and B. T. Carr. 2006. Sensory Evaluation Techniques. 4th ed. CRC Press, Boca Raton, FL.

Menis, M. E. C., T. M. G. Milani, A. J. M. Boscolo, and A. C. ContiSilva. 2013. Extrusion of flavored corn grits: Structural characteristics, volatile compounds retention and sensory acceptability. LWT Food Sci. Technol. (Campinas) 54:434-439.

Morais, E. C., A. R. Morais, A. G. Cruz, and H. M. A. Bolini. 2014. Development of chocolate dairy dessert with addition of prebiotics and replacement of sucrose with different high-intensity sweeteners. J. Dairy Sci. 97:2600-2609.

Moskowitz, H. R., and J. Hartmann. 2008. Consumer research: Creating a solid base for innovative strategies. Trends Food Sci. Technol. 19:581-589.

Paixão, J. A., J. B. Rodrigues, E. A. Esmerino, A. G. Cruz, and H. M. A. Bolini. 2014. Influence of temperature and fat content on ideal sucrose concentration, sweetening power, and sweetness equivalence of different sweeteners in chocolate milk beverage. J. Dairy Sci. 97:7344-7353.

Popper, R., W. Rosenstock, M. Schraidt, and J. B. Kroll. 2004. The effect of attribute questions on overall liking ratings. Food Qual. Prefer. 15:853-858.

Prescott, J. 2015. Multisensory processes in flavour perception and their influence on food choice. Current Opinion Food Sci. 3:47-52.

Prudencio, I. D., E. S. Prudencio, E. F. Gris, T. Romazi, and M. T. Bordignon-Luiz. 2008. Petit Suisse manufactured with cheese whey retentate and application of betalains and anthocyanins. LWT Food Sci. Technol. (Campinas) 41:905-910.

Ramírez-Santiago, C., C. Lobato-Calleros, H. Espinosa-Andrews, and J. E. Vernon-Carter. 2012. Viscoelastic properties and overall sensory acceptability of reduced-fat Petit-Suisse cheese made by replacing milk fat with complex coacervate. Dairy Sci. Technol. 92:383-398.

Small, D. M. 2012. Flavor is in the brain. Physiol. Behav. 107:540-552

Soukoulis, C., I. Chindrinos, and C. Tzia. 2008. Study of the functionality of selected hydrocolloids and their blends with kappacarrageenan on storage quality of vanilla ice cream. LWT Food Sci. Technol. (Campinas) 41:1816-1827.

Soukoulis, C., and C. Tzia. 2009. Response surface mapping of the sensory characteristics and acceptability of chocolate ice cream containing alternate sweetening agents. J. Sens. Stud. 25:50-75.

Spence, C. 2015. Multisensory flavor perception. Cell 161:24-35.

Stone, H., R. N. Bleibaum, and H. A. Thomas. 2012. Sensory Evaluations Practices. 4th ed. Academic Press, New York, NY

Tournier, C., C. Sulmont-Rossé, E. Sémon, A. Vignon, S. Issanchou, and E. Guichard. 2009. A study on texture-taste-aroma interactions: Physico-chemical and cognitive mechanisms. Int. Dairy J. 19:450-458.

Van Kleef, E. 2006. Consumer research in the early stages of new product development. $\mathrm{PhD}$ thesis. Wageningen University, Wageningen, the Netherlands.

Vickers, Z. 1988. Sensory specific satiety in lemonade using a just right scale for sweetness. J. Sens. Stud. 3:1-8. 